\title{
Schwierigkeiten emigrierter Frauen in Brasilien: Eine autobiographische Studie
}

\section{Katherine Morris}

Erleben und beschreiben Frauen historische Momente anders als Männer? Entlehnen sie einfach stilistische und thematische Elemente, die sie gewöhnlich in Autobiographien von Männern gefunden haben, oder haben sie ihr eigenes Genre geschaffen? Ich will versuchen, diese und andere Fragen anhand einer Fallstudie über deutsch-jüdische Frauen zu beantworten, die während der größten Wirren des 20. Jahrhunderts nach Brasilien einwanderten: im Zweiten Weltkrieg sowie unmittelbar vorher und danach. ${ }^{1}$

Viele Kulturhistoriker betrachten heute die Bedeutung des Geschlechts in Literatur und Geschichte mit anderen Augen, desgleichen auch die Art und Weise, wie außerordentliche Ereignisse gewöhnliche Menschen berühren. Die Gewalttaten des Zweiten Weltkrieges, die Transit, Exil, Holocaust und Einwanderung bestimmten, veränderten das Leben aller davon Betroffenen, aber man könnte nicht behaupten, daß sie Männer und Frauen auf gleiche Weise berührten. Die kulturelle Sphäre war für Frauen stets eine andere als die für Männer, und diese Feststellung ist nicht weniger zutreffend im Falle von Wendepunkten in der Geschichte. Gerade die Art und Weise, in der deutschjüdische Frauen die wichtigsten gewaltsamen Veränderungen dieses Jahrhunderts in Erinnerung behielten, liefert uns einen wichtigen Aspekt für das Verständnis dieser Ära, und hier wiederum besonders für den Exodus nach Südamerika, nach Brasilien.

Die Autobiographien von Frauen beschreiben und erforschen globale Fragen des Exils und der räumlichen Veränderung, aber wenige Studien zielen auf eine methodologische Basis für ein solches Unterfangen. Autobiographie als Quelle führt zu der Frage nach der historischen Echtheit von "Literatur". Und dies ist auch der Kern des Problems: Sollten Autobiographien aus dieser turbulenten Zeit als Literatur oder als Geschichtsschreibung bewertet werden, oder als beides - Fakten und Fiktion? Wie kann eine Methodologie für geschlechtsbezogene Studien über Exil- und Holocaustmemoiren formuliert werden? Es gibt nur wenige Präzedenzfälle, die bei der Untersuchung der Exil-Autobiographie von Frauen hilfreich sein könnten. Viele Kritiker neigen dazu, Autobiographien als Literatur zu betrachten und tun sich oftmals schwer mit dem Thema Literatur als Geschichtsschreibung. Neuere Veröffentlichungen wie Heinemanns Gender and Destiny und Youngs Writing and Rewriting the Holocaust widmen

${ }^{1}$ Zur politischen Situation Brasiliens in diesen Jahren, die den Hintergrund der individuellen Schicksale bildete, siehe den Beitrag von Izabela $\mathrm{M}^{\mathrm{a}}$ Furtado Kestler in diesem Band. 
sich dem Problem der Interpretation hinsichtlich des Holocaust, aber keiner dieser Texte lenkt den Blick auf das Exil.

Die feministische Kritik hat unsere Perspektiven der Definition von Literatur erweitert und die Erforschung der Literatur und Geschichte von Frauen vertieft. Aufgrund der Fortschritte im Bereich der feministischen Kritik hinsichtlich dessen, was nicht als "Literatur" im traditionellen Sinne betrachtet wurde, werden neue Klassifikationen vorgenommen und erörtert ${ }^{2}$. Mein Beitrag reflektiert dieses Interesse an geschlechtsspezifischen Untersuchungen, indem er sich den Exilerfahrungen von Frauen zuwendet. Er ist eine kulturgeschichtliche Studie vom Standpunkt einer Frau aus. Die Frauen im brasilianischen Exil kämpften nicht auf Schlachtfeldern, noch zogen sie neue Grenzen als Politikerinnen. Ich untersuche die Geschichte aus der Perspektive "gewöhnlicher" Frauen, die keine Generäle waren, sondern Bürgerinnen und Opfer. Mein Beitrag untersucht das Exil, wie es durch die Augen einiger deutsch-jüdischer Frauen gesehen wurde, die in den 1930er Jahren aus Nazi-Deutschland flohen und nach Brasilien einwanderten. ${ }^{3}$

Die herausragenden Charakteristika dieser Autobiographien waren der Kontext des Zweiten Weltkrieges und seiner Folgen, die politische Marginalisierung der deutsch-jüdischen Fragen sowie das literarische Genre, in dem sie ihre Lage als deutsch-jüdische Frauen in Hitlers Drittem Reich auszudrücken pflegten (Personal Narratives Group 1989). Die vielfältigen Beziehungen zwischen Verfasserinnen und Erzählerinnen kommen dabei ebenso ins Spiel. Die nachstehenden Ausführungen stellen eine kurze Diskussion der Hauptfragen meiner Arbeit dar: die Marginalisierung, die Vielfalt des Genres und die kulturellen Aspekte von Autobiographien.

Die Rolle der jüdischen Frau in Hitlers Drittem Reich war politisch marginal in Beziehung zur herrschenden Klasse jener Zeit. Nicht nur war sie infolge ihres Judentums fern von jeder Form von Einfluß in der Gesellschaft, sondern auch infolge ihres Frauseins. Es ist wichtig, zur Kenntnis zu nehmen, wie diese Frauen sich selbst betrachteten und ob sie sich selbst als marginal ansahen. Irene Gruenbaum war sich dessen voll bewußt und erwähnte es gleich auf ihrer ersten Seite:

Hitler hatte über das Schicksal von Millionen Menschen entschieden, auch über mein Schicksal, weil ich Jüdin bin, Kind jüdischer Eltern und mit einem Juden verheiratet. ${ }^{4}$

Das Genre dieser Texte beschreibt den Rahmen, den die Frauen bei der Erzählung ihres Lebens zu verwenden pflegten. War dies die Nachahmung einer männlichen Autobiographie? Was betrachtete man als "normales" Leben oder

\footnotetext{
${ }^{2}$ Benstock 1988, Heilbrun 1988, Smith 1987.

${ }^{3}$ Bridenthal/Grossmann/Kaplan 1984, Lixl-Purcell 1988.

${ }^{4}$ Das Zitat ist dem unveröffentlichten Manuskript entnommen.
} 
als "normale" Geschichte? Der Hintergrund dieser Frauen war im wesentlichen ähnlich, insofern sie alle der deutsch-jüdischen Mittelschicht angehörten, als sie ihre Texte schrieben, und sie waren sich des Genres der Autobiographie voll bewußt, obwohl die meisten diesen Begriff zur Kennzeichnung ihres Schreibens nicht verwenden würden. Alle wußten, daß sie ungewöhnliche Erfahrungen durchmachten, weil sie Juden in Hitlers Deutschland waren. Ihr Schreiben erlaubte es ihnen, den vergangenen Erfahrungen einen Sinn abzugewinnen und sie in einen zusammenhängenden Bericht einzufügen. Mit diesem Vorgang organisierten und interpretierten sie ihr Leben und gaben ihm eine neue Bedeutung.

\section{Marthe Brill und Alice Brill Czapski}

Marthe Brill wurde 1894 in Köln geboren und entstammte einer Familie intellektueller Frauen. Ihre Mutter, Bertha Leiser, war Schriftstellerin und Vorkämpferin für Frauenrechte. Beide Eltern waren Juden, aber nachdem sie als Kind ihre Mutter verloren hatte, wurde sie nicht religiös erzogen. Marthe Brill war eine liberal gesonnene Intellektuelle und interessierte sich für alles, was mit Judentum zu tun hatte, von der Geschichte bis zur Mystik. Sie erwarb ihren Doktorgrad in Wirtschaftswissenschaften kurz vor dem Ersten Weltkrieg und arbeitete während des Krieges für das Weltwirtschaftsinstitut in Hamburg. Wegen ihres Interesses für Literatur und Journalismus schrieb sie später Artikel, Kurzgeschichten und Reiseberichte für Zeitschriften und arbeitete als Korrespondentin für das Hamburger Fremdenblatt.

Der Schmelztiegel ist ein autobiographischer Roman, in dem Marthe Brill über ihre Wanderjahre zwischen 1933 und 1939 berichtet. Sie erzählt die Geschichte von Sylvia und ihrer Tochter Miriam in der Art eines Schlüsselromans. Das war zu jener Zeit in Mode und gab der Autorin freie Hand in der Beschreibung von Menschen, ohne ihre wahre Identität im Falle einer Veröffentlichung preisgeben zu müssen. Durch die Verwendung dieses Genres definiert die Autorin die Autobiographie sowohl als erzählerisches wie als historisches Werk. Obwohl Marthe Brills Roman der Realität entspricht, schreibt sie in der dritten Person und verschafft sich dadurch sichere Distanz zu einer tragischen und schwierigen Zeit in ihrem Leben. Ihr Text Der Schmelztiegel ist chronologisch geschrieben; er beginnt mit ihrem Leben in Hamburg 1933 und endet mit ihrem Leben in Brasilien.

Ihre Tochter Alice Brill Czapski lebt heute in São Paulo; sie hat ebenfalls Memoiren unter dem Titel Memories from 1933-1945 verfaßt. In diesen Memoiren bezieht sie sich auf das Tagebuch, das sie als Zwölfjährige begonnen hatte und zitiert daraus. Alice Brill wird hier erwähnt, weil ihre Autobiographie zwei Gesichtspunkte vereint: den ihrer gegenwärtigen Person als reife Frau, und den der jungen Alice Brill, die als Mädchen ein Tagebuch geschrieben hatte. Sie erscheint im Roman ihrer Mutter - Der Schmelztiegel - auch als Sylvias Tochter Miriam. Auf diese Weise haben wir drei verschiedene Quellen, die ihr Leben im Transit und ihre frühen Jahre in Brasilien beschreiben. 
Sylvias Roman Der Schmelztiegel ist die Geschichte einer deutschjüdischen Flüchtlingsfrau und der Unbillen, die sie erleidet. Sie war Sekretärin des neugegründeten Hilfskomitees für jüdische Emigranten. Sie wollte Emigranten, die Deutschland meist ohne Geld verließen, Obdach und Arbeit besorgen. Sie waren verängstigt, sprachen kein Portugiesisch, und wenige hatten einen praktischen Beruf. Da sie selbst Flüchtling war, brachte sie Mitgefühl für Menschen in ähnlicher Situation auf und wußte, daß sie sich den neuen Verhältnissen anpassen mußten, um zu überleben.

In endloser Reihe kamen die Emigranten in ihr Büro und suchten Obdach und Arbeit:

Es war traurig und tröstend zugleich: traurig, daß die Macht zu helfen so beschämend gering war. Helfen, das fühlte Sylvia bald, konnte man immer nur denen, die sich selbst helfen wollten, und die zum Äußersten bereit waren. [...] Frauen allein, das sah Sylvia mit Erstaunen, waren am leichtesten unterzubringen; sie fanden Arbeit in Häusern und Kontoren, als Wirtschafterinnen, Erzieherinnen, Stenotypistinnen. Es gab auf diesem Kontinent noch nicht allzu viele Frauen, die arbeiteten, und sie wurden gebraucht. (Brill 1939, 193).

Alice Brill Czapski erwähnt in ihrer Autobiographie gleichfalls die Arbeit ihrer Mutter. Sie erzählt von den Schwierigkeiten Marthe Brills bei der Hilfsarbeit für Flüchtlinge. Frauen scheinen sich leichter als Männer an die Emigration angepaßt zu haben - sowohl mental als auch praktisch. Ihre Tochter schreibt dazu in ihrer Autobiographie: "Meine Mutter versuchte ihr Bestes, ihnen zu helfen. Einige waren erfinderisch und wurden mit den Schwierigkeiten des Überlebens fertig, vor allem Frauen. Frau Reichmann zum Beispiel. Sie war die Ehefrau eines Gelehrten und Mutter eines zehnjährigen Jungen. Sie entschloß sich, eine Pension zu eröffnen, mietete ein nettes Haus und begann zu kochen, zu putzen und Wäsche für ihre Gäste zu waschen. Meine Mutter war ihr erster Logiergast, sie war froh darüber, einen Raum für uns zwei zu haben und sich nicht um die Mahlzeiten kümmern zu müssen" (Brill Czapski 1991).

Marthe Brill und ihre Tochter Alice kontrastieren scharf mit Erich Brill. Marthe Brill heiratete Erich Brill 1920 und ließ sich 1922, als Alice geboren wurde, von ihm scheiden. Er war Künstler und an die kosmopolitische Atmosphäre Europas gewöhnt; 1934 gelangte er mit seiner Tochter nach São Paulo. Er blieb für einige Monate in Brasilien und schloß Freundschaft mit anderen Künstlern, konnte sich aber nicht an das neue Land gewöhnen. Es bot nicht die gleiche kulturelle Tradition wie Deutschland, und er war ruhelos, unglücklich und in politischen Fragen naiv. Eines Tages verkündete er, daß er im Januar 1936 nach Deutschland zurückkehren werde. Marthe Brill versuchte vergebens, ihn davon abzubringen. Sie sagte ihm, daß zurückkehrende Flüchtlinge eingesperrt würden, aber er glaubte ihr nicht. "Die Deutschen sind unfähig zu solchem Verhalten, es ist nicht wahr, sie sind ein zivilisiertes Volk", recht- 
fertigte er sich. Nichts konnte ihn davon abbringen. Er sagte einfach: "Ich vertraue meinem Schicksal". Alice Brill stellt in ihrer Autobiographie fest: "Er hörte niemals auf, an mich zu denken. Ich lehnte mich auf, ich war damals 16 Jahre alt" (Brill Czapski 1991, 11).

Als er in Hamburg ankam, nahm niemand von ihm Notiz. Er genoß Monate der Freiheit, in denen er durch Deutschland und Europa reiste und malte, wo immer er hinging. Aber seine Zeit lief ab. Alice Brill stellt fest: "Ich denke, $\mathrm{da} ß$ er es schließlich geahnt haben muß, denn sein letztes Selbstportrait, das er an der Jahreswende 1936/37 gemalt hat, gibt mir immer wieder ein Gefühl, das mich an das Bildnis des Dorian Gray erinnert" (Brill Czapski 1991, 13). Er wurde bald unter der Anklage der "Rassenschande" verhaftet, weil er eine "arische" Freundin habe; im Verlauf des Prozesses gestand er, daß er das Gesetz mißachtet habe. Erich Brill verbrachte ein Jahr im Gefängnis und wurde dann zu fünf Jahren Zwangsarbeit verurteilt. Im Jahre 1946 erfuhr Alice vom Schicksal ihres Vaters. Ihre Tante erhielt auf eine Anzeige im Aufbau eine Antwort mit der Bestätigung, daß er nach Entlassung aus dem Gefängnis einem Transport nach Riga zugewiesen und am 26. März 1942 mit Tausenden von anderen hingerichtet worden war.

Im Roman Der Schmelztiegel führt Marthe Brill ihren früheren Mann als Maler Erich Schönberg ein. Sie schildert, wie der Nationalsozialismus die Künstler und Denker sogar in Brasilien berührte. Erich war ein junger deutscher Jude, der ganze Koffer mit Leinwand aus Europa mitbrachte, ohne sich in São Paulo zu vergegenwärtigen, daß er diesen Lebensstil in Brasilien kaum würde halten können:

Sein Traum erfüllte sich: Auf der Avenida Rio Branco, mitten im Getriebe der Millionenstadt, stellte er seine Bilder aus, in den Räumen eines deutschen Clubs. Eine Gruppe von Nationalsozialisten versuchte seine Ausstellung zu sprengen. Er wehrte sich; er kämpfte um sein Recht. 'Ich bin Deutscher', erklärte er zornig, 'ich habe für Deutschland geblutet; meine Bilder hängen noch heute unangetastet in den glänzendsten Museen meiner Heimat...'. Er setzte seinen Willen durch. Empfänge, Cocktail-Parties, blendende Kritiken; der Ruhm eines Tages [...] Kein Bild wurde verkauft: die Deutschen kauften nicht, weil er Jude war, und die Juden mieden seine Ausstellung, weil sie in einem deutschen Club standfand. Wovon sollte der Maler leben? (Brill 1939, 231).

\section{Mirjam Logat}

Wie sich diese deutsch-jüdischen Frauen akklimatisierten, bildet den Gegenstand des Textes von Mirjam Logat. Sie wählte für ihre Beschreibung von Exil und Einwanderung die Form einer Erzählung unter dem Titel Ich bin ausgewandert. Ihr Bericht beginnt mit dem antijüdischen Boykott vom 1. April 1933 und führt fort über ihre Auswanderung nach Brasilien bis zu ihrem Leben in 
São Paulo. Wie Marthe Brill berichtet sie in der dritten Person. Jedoch schreibt sie - anders als viele andere Verfasserinnen von Autobiographien - unter ihrem Schriftstellernamen Mirjam Logat. In Wirklichkeit hieß sie Martha Glogauer. Sie ging im Dezember 1933 mit ihrem Ehemann und einer kleinen Tochter nach São Paulo, nachdem ihr Mann aus seiner guten Stellung bei der AEG entlassen worden war. Er blieb sein ganzes Leben krank und arbeitslos. Mirjam Logat schrieb das Manuskript für die ganze Familie.

Wir können annehmen, daß eines der Motive ihres Schreibens in dem Wunsch bestand, die für alle jüdischen Flüchtlinge charakteristischen Probleme herauszustellen: "Es ist das Leben der ausgewanderten Frau; zwar die Geschichte einer Einzigen, eingezwängt im ganz Persönlichen, doch gleichzeitig das Gemeinschaftsschicksal aller Frauen in der Umschichtung der Emigration" (Logat 1990).

Die Verfasserin stellt fest, daß alle Frauen, die nach Brasilien ausgewandert waren, ähnliche Probleme hatten und sie auf individuelle Weise lösten. Aber vielleicht ist der interessanteste Teil ihrer Einführung die Art, in der sie die Handhabung ihres Genres beschreibt: "Alle Namen, soweit sie überhaupt zitiert sind, sind frei erfunden, und irgendwelche Identität wäre reiner Zufall. Die Geschehnisse beruhen jedoch auf absoluten Tatsachen" (Logat 1990, 1). Wiederholt werden fiktive Namen genannt, um Personen zu charakterisieren und Ereignisse zu berichten, die tatsächlich stattgefunden haben. Dies schützt die Verfasserin davor, reale Personen preiszugeben.

Trotz der fiktiven Namen sind die Fakten real. Statt des autobiographischen "Ich" verwendet sie die dritte Person und erzählt ihre Geschichte, indem sie sich auf Marion als Hauptperson konzentriert. Es ist schwer zu entscheiden, inwieweit sich die Autorin in der Figur Marions selbst porträtiert hat. Wichtiger jedoch ist die Stilisierung Marions zu dem typischen Schicksal einer jüdischen Frau, die die ihr völlig neuen Lebensumstände in Brasilien bewältigen muß.

Mirjam Logat spricht von einer jüdischen Hilfsorganisation, die Marion und ihren Ehemann Peter anfangs unterstützte. Schrittweise lernte Marion Portugiesisch, wurde unabhängiger und eröffnete eine Pension für jüdische Einwanderer. Sie hatte deutliche Vorstellungen von der Rolle der Frauen in dieser Einwanderungswelle:

Die Frauen fanden irgendeinen Weg nach vorne; sie arbeiteten, sie schufteten, sie opferten sich auf, sie verteidigten sich selbst und stützten die Familie. Marion, die so vieles sah, hörte und erlebte, wußte ganz genau, daß sie selbst kein Einzelschicksal war, nur eine von all den vielen, die ausgewandert ein neues Ziel erkämpften. Sie gehörte lediglich zu den Ungezählten, die mit festem Willen und eiserner Energie vorwärtsstrebten und durchhielten (Logat 1990, 34).

Auffällig ist die Charakterisierung Marions als eine der "ungezählten", ein Ausdruck, der ihre Wichtigkeit herunterspielen soll. Ungeachtet der Tatsache, 
daß sie viele der Hindernisse gemeistert hat, denen emigrierte Frauen gegenüberstanden, war sie eine der "ungezählten" anderen in Brasilien, die sich an ein neues Land und eine neue Kultur angepaßt hatten. Viele dieser Frauen hatten Erfolg, aber sie blieben unbemerkt am Rande der patriarchalischen Kultur der Dritten Welt. Der Charakter Marions verleiht dem ihres Mannes Konturen. Peter verkümmerte in Brasilien:

Sein Selbstvertrauen schwand immer mehr, seine Energie versagte vollkommen, die Hände zitterten, die Nerven streikten, die Flucht in die Krankheit wurde zur Entschuldigung, zur Abwehr der Verantwortung - und steigerte sich zur völligen Gleichgültigkeit für Umwelt und Geschehen. Ein einfacher Bronchialkatarrh wurde fast zur tödlichen Krankheit aus Mangel an Lebenswillen und Lebenskraft. Jahrzehnte taumelte er in diesem Zustand von krank und gesund. Selbstverständlich und zwangsweise übernahm es Marion, das Geschick der Familie zu steuern (Logat 1990, 25).

Frauen waren Teil dieser Gruppe von namenlosen Personen, die schrittweise ihren Weg in einer Gesellschaft machten, die ein Schmelztiegel von verschiedenen Rassen und Religionen war. Frauen, die in den 1930er und 1940er Jahren in Brasilien lebten, waren kein Teil einer männlichen Übermacht und hatten unter anderen Umständen zu leben als diejenigen, die an europäische Verhältnisse gewöhnt waren.

\section{Hilde Wiedemann}

Hilde Wiedemann schrieb ihre Autobiographie Der Weg ist das Ziel im Jahre 1966 und war sich dabei des Genres und des Schreibvorganges bewußt. Ihre erzählerische Form ist stärker traditionell. Sie beginnt mit der Beschreibung ihrer Umgebung während des Schreibvorganges im Hause einer deutschen Kolonie im brasilianischen Bundesstaat Paraná: "Die Schreibstube ist klein, der Schreibtisch in die Ecke rechts vom Fenster eingebaut. [...] Ich öffne den Aktenschrank und lege mir vorsichtig ein paar Familiendokumente auf den Schreibtisch..." (1979, Kap.1). Hilde Wiedemann ist hochgebildet und beginnt eine ungewöhnliche Geschichte auf gewöhnliche Weise. Sie beginnt nicht damit, wie sie in den 30er Jahren im brasilianischen Urwald einen Bauernhof betreibt, sondern mit der Geschichte ihrer Großeltern in Deutschland. Ihre Autobiographie geht in chronologischer Reihenfolge vor: Sie setzt an mit ihrer Ankunft in Santos 1933 und schildert dann ihre Eindrücke von São Paulo, ihr Leben auf einem Bauernhof in Terra Nova/Paraná im Süden und in Recife/Pernambuco an der Nordostküste. Sie liefert ebenso Beschreibungen von verschiedenen Plätzen in Brasilien, an denen sie mit ihrer Familie Ferien machte. Manche Teile lesen sich wie ein Reisebericht über Brasilien in einer Zeit, in der die meisten Landschaften noch unberührt waren. Aber Hilde Wiedemann erörtert auch die Stellung der Frau und die politische Lage der Deutschen und der Juden während der Kriegsjahre. 
1933 war São Paulo noch eine typisch brasilianische Stadt, noch nicht die auswuchernde Metropole von heute. Die allgemeine Haltung gegenüber Frauen in der Gesellschaft war eher provinziell und völlig anders als die vorherrschende Haltung im Europa jener Zeit:

Was mir vor allen Dingen auffiel, war das überwiegend von Männern bestimmte Straßenbild. An allen Ecken standen Gruppen plaudernder Männer: An den Theken der nach der Straße hin offenen Cafés drängten sie sich ebenso wie an kleinen Marmortischen, wo bei einigen Tassen Mokka anscheinend die Geschäfte des Tages abgeschlossen wurden. Eine einzelne Frau als einfache Passantin schien durchaus Seltenheitswert zu besitzen, jedenfalls den ungenierten Blicken nach zu urteilen, die mir und dem Kind von den Straßenecken aus folgten (Wiedemann 1979, Kap. 24).

Sie verbrachte fast sieben Jahre auf einem Gehöft in einer deutschen Kolonie nahe Castro in Paraná. Ihr schwieriges Pionierleben auf der Farm in Terra Nova endete 1939 mit einem Umzug nach Curitiba, der Hauptstadt des Bundesstaates Paraná. Dort gab es ein eigenartiges Nebeneinander von deutschen Nazis und jüdischen Einwanderern, das typisch für Südbrasilien war.

Brasilien bot vielen Nazi-Gegnern Asyl, und Hilde Wiedemann war eine von vielen deutschen Juden, die in das größte Land Südamerikas auswanderten. Brasilien nahm in den 30er Jahren 15.000 deutsche Juden auf. 1941 hielten sich 20.000 deutsch-jüdische Emigranten im Lande auf. ${ }^{5}$ Zur gleichen Zeit lebten dort aber zahlreiche Deutsche, die mit dem Nationalsozialismus sympathisierten:

In Curitiba war das Fortschreiten der Nazifizierung aber wesentlich deutlicher fühlbar. Deutsche gehörten dort entweder der Partei an, dann waren sie 'gute Deutsche', oder sie waren Juden, und dann existierten sie gar nicht. Es gab aber auch noch einige wenige, die weder Parteimitglieder noch Juden waren. Sie behaupteten, Demokraten zu sein und ihre Meinungen frei äußern zu dürfen, weshalb sie für leicht idiotische Sonderlinge gehalten wurden (Wiedemann 1979, Kap. 42).

Das Bild der deutschen Juden und der Nazi-Sympathisanten wurde noch komplizierter durch die antisemitische Politik des Präsidenten Getúlio Vargas. Sein "Estado Novo" drehte sich um einen brasilianischen Nationalismus und wurde gegenüber Juden immer restriktiver. Diese sonderbare Mischung ist vor dem Hintergrund der sich verschärfenden Politik der Einwanderungsbeschränkung

\footnotetext{
XXXIV.

${ }^{5}$ Frühwald/Schieder 1981, Kießling 1981, Biographisches Handbuch 1983, II: XXII,
} 
gegen die große Zahl der in den 1930er Jahren meist aus Deutschland kommenden Juden zu begreifen (Lesser 1989, 219).

Als der Krieg ausbrach, froren die Beziehungen zwischen Brasilien und Deutschland ein, wodurch das Schicksal von Hilde Wiedemann und ihrer Familie unsicher wurde. Sie zogen nordwärts in das tropische Recife, wo sie zwanzig Jahre lang blieben. Selbst in dieser Stadt konnte man die Folgen des Krieges erkennen. Alle deutschen Männer in der Stadt wurden eingesperrt, ob sie nun Sympathisanten oder Gegner des Nationalsozialismus waren. In den ersten Kriegsjahren wurden in allen Bundesstaaten Internierungslager für die Angehörigen der Achse eingerichtet. Die politisch stärker aktiven Männer wie Funktionäre der NSDAP oder der SS wurden auf einer kleinen Insel in der Bucht von Rio gefangengehalten (Wiedemann 1979, Kap. 48).

So wie der Krieg die politische Situation in Brasilien änderte, hatte er auch Auswirkungen auf die soziale Lage. Fremde Truppen vermittelten den Leuten stärker westliche Ideen. Hilde Wiedemann zeichnet ein Bild dessen, was damals (1940) von Frauen in Brasilien erwartet wurde, und vermerkt ihren marginalen Status. Sie reflektiert ihre Erfahrungen als Frau in Recife und kommentiert die verschiedenen Rollen von Männern und Frauen:

Wenn Wilhelm und ich zusammen eingeladen waren, wurde es mir anfangs immer schwer, mich brav und ausschließlich zu den weiblichen Gästen zu halten, denn es herrschte noch strenge Scheidung der Geschlechter. Die Männer standen herum oder fanden einen kühlen Sitzplatz im Garten, wo sie sich über Geschäfte oder Politik unterhielten. Wir Frauen saßen auf den Sofas und in den Lehnstühlen und besprachen Krankheiten, Dienstboten und Kinder (Kap. 47).

Aber diese Trennung nach Geschlechtern änderte sich schrittweise während des Krieges: "Die entscheidende Wendung in Richtung Gleichschaltung der Geschlechter brachte der Krieg". Brasilien stand auf seiten der Alliierten, und amerikanische Truppen wurden dort stationiert. Recife war Aufenthaltsort für 20.000 amerikanische Marinesoldaten, die den "American way of life" dorthin brachten. Dieser Wandel des Lebensstils begann, auf die Einheimischen abzufärben:

Die Amerikanerinnen machten nicht nur ihre Einkäufe unbegleitet, sie gingen sogar allein ins Kino oder ruhten sich bei einer Portion Speiseeis an einem Kaffeehaustischchen aus, als einzige Eskorte einen Beutel voll Paketen, die sie später noch höchst eigenhändig nach Hause trugen (Kap. 49).

Von ihrem Umfeld her steht Hilde Wiedemann in einer ganz anderen Umgebung als die Auswanderer, die in die USA oder in europäische Länder gingen. Sie war Frau in einem Drittweltland. Wie viele andere deutsch-jüdische Frauen im Exil war Hilde Wiedemann politisch klug. Dieses Bewußtsein war sicherlich 
geprägt durch die außerordentlichen Umstände, die sie in Deutschland und Brasilien erfahren hatte. Sie reflektiert nachdenklich über die Kriegsjahre, über Nazi-Deutschland und über die Zukunft von Brasilien. Obwohl die Deutschen in Brasilien nicht durch den Krieg isoliert waren, begriffen keineswegs alle die vollen Konsequenzen, als er vorüber war. Hilde Wiedemann beschreibt im Detail, wie sie am Ende des Krieges von der Zerstörung Deutschlands und von der Vernichtung der Juden hörte:

$\mathrm{Zu}$ der Trauer über das, was an kulturellen Schätzen für immer verloren war, kam das Entsetzen über die Scheußlichkeiten, zu denen unser Volk, das wir immer als anständig und ehrlich geliebt hatten, sich hatte herabwürdigen lassen (Kap. 50).

\section{Trudi Landau}

Trudi Landau, geb. Joseph, wurde am 2. Mai 1920 in Köln geboren. 1927 zog ihre Familie nach Opladen um und blieb dort bis Ende 1938. Trudi Joseph war erst dreizehn, als Hitler an die Macht kam, und der Krieg zwang sie, Opladen zu verlassen. Sie lebte im Exil in Belgien und Frankreich bis zum Ende des Krieges und schiffte sich im November 1945 nach Brasilien ein. Wäre sie zu dieser Zeit unverheiratet gewesen, stellt sie fest, dann wäre sie nach Palästina gegangen, um zu ihrer Mutter zu ziehen. Sie ging nach Brasilien, weil ihre Schwiegereltern sich dort schon 1936 niedergelassen hatten. Die Landaus schickten Trudi und ihrem Ehemann die beantragten Dokumente und Aufenthaltsgenehmigungen. Sie erreichten den Hafen von Santos am 31. Dezember 1945.

Eines der Hauptthemen ihres Schreibens ist die Bestimmung ihrer Identität als Deutsche und als Jüdin. Wie viele deutsch-jüdische Einwanderer in Brasilien fiel ihr die Akklimatisierung anfangs schwer. Da sie kein Portugiesisch verstand und keine Arbeit finden konnte, war sie gezwungen, im Hause ihrer Schwiegereltern zu bleiben. Überdies hatte sie ein kleines Baby, das im Februar 1946 geboren worden war. "Mit einem Baby, ohne die Landessprache zu sprechen oder die Stadt zu kennen oder irgendeinen von meinen Bekannten um mich zu haben, fühlte ich mich schrecklich isoliert" ${ }^{6}$. Da sie Jahre im Exil oder Transit in Europa verbracht hatte und zwangsweise auf eigenen Füßen hatte stehen müssen, hatten sich viele ihrer Ideen und Gewohnheiten geändert. Der Unterschied zwischen der alten und der neuen Generation wird am besten illustriert durch das Verhalten ihrer Schwiegermutter:

Eines Nachts, als ich in den Baderaum ging, sah sie, daß ich in der tropischen Hitze kein Nachthemd trug; sie wurde grob und sagte mir, daß ihr Mann sie niemals nackt gesehen habe. [...] Meine Gefühle müssen auf gewisse Weise dieselben gewesen sein wie die von Soldaten, wenn sie nach dem Kriege nach Hause kommen. Sie sehen, daß

${ }^{6}$ Die Zitate sind dem unveröffentlichten Manuskript entnommen. 
das Leben daheim weitergegangen ist, mit denselben Frivolitäten und Konventionen. Die, die zu Hause geblieben sind und nicht ihre ganze Existenz abgeschüttelt haben, können nicht verstehen, daß Soldaten plötzlich andere Präferenzen haben und anders urteilen (22).

Von besonderem Interesse ist hier ihre Identifikation mit heimkehrenden Soldaten. Dies unterstreicht ihre Gefühle von Marginalisierung, weil sie Dinge anders betrachtet als nach der allgemein akzeptierten Norm. Obwohl sie nicht Soldat in Europa gewesen war, war sie als Jüdin, die Nazi-Deutschland entkommen war, zahlreichen Gefahren ausgesetzt gewesen.

Anfangs waren sie und ihr Mann finanziell vollkommen abhängig von den Schwiegereltern. Es war schwierig für sie, vor allem nachdem sie für eine so lange Zeit Flüchtling in Europa gewesen war:

Als ich nach Brasilien kam, fühlte ich, daß alle anderen in Frieden gelebt hatten, während ich litt, geliebte Personen verlor und Härten ausgesetzt war. Ich fühlte, daß jeder mir etwas 'schuldete', mindestens in Form von Toleranz (Landau 1990, 23).

Schrittweise besserte sich ihre finanzielle Lage, und ein Jahr später zogen sie um nach Bom Retiro, einem jüdischen Viertel von São Paulo, das früher von osteuropäischen Juden, die den Pogromen entflohen waren, bevölkert worden war. Ab 1933 ließen sich auch die vor Hitler fliehenden deutschen Juden in Bom Retiro nieder.

Trudi Landau wurde in Brasilien zunächst als Sekretärin einer Schweizer Firma angestellt, wo sie in deutscher Sprache korrespondierte. Danach arbeitete sie für eine belgische Firma, für die sie französischen Briefwechsel erledigte. Da sie mehrsprachig war, suchte sie sich sodann eine Stelle, in der sie ihr Englisch gebrauchen konnte. Im allgemeinen waren solche Anstellungen mit höheren Gehältern verbunden. 1952 begann sie, bei Braniff International Airways als Sekretärin des Distriktmanagers zu arbeiten. Auf diese Weise verdiente sie Geld für die Familie und lernte an ihrem Arbeitsplatz auch schrittweise Portugiesisch.

$\mathrm{Zu}$ Beginn war sie umgeben von der jüdischen Gemeinschaft in Bom Retiro, so daß sie ihren Sohn in einen jüdischen Kindergarten schickte. Sie erzählt, wie es in jener frühen Zeit zuging: "Ich hatte niemals Zeit für ein müßiges Gespräch, weil ich die meiste Zeit in der Arbeit war, lange Zeit auch sonnabends bis ein Uhr" (Landau 1990, 25a). Viele deutsch-jüdische Frauen in Brasilien arbeiteten in Büros und begannen auf diese Weise ein neues Leben, weil Frauen für diese Arbeit gebraucht wurden. Sie war viele Jahre als Schriftstellerin tätig und hat stets die Menschenrechte verteidigt. Nicht nur hat sie die Schrecken der Nazi-Zeit auf sehr persönliche Weise erlebt, sondern auch über politische Verfolgungen in Brasilien in der Zeit der Militärdiktatur (1964-79) geschrieben. Ihr Buch Vlado Herzog. O que faltava contar schildert das Leben eines jüdischen Journalisten, der von der Diktatur gefoltert und ermordet wurde. 


\section{Käte Kaphan}

1936 emigrierte Käte Kaphan aus Nazi-Deutschland und landete mit ihrem Ehemann und drei Kindern im Hafen von Santos. Sie schrieb ihren Text zwanzig Jahre später unter dem Titel Immigration into the Brazilian Jungle im Jahre 1956. Im ersten Absatz stellt sie fest:

Was auch immer unser Schicksal uns bringen mochte, wir wollten die Vergangenheit vergessen, denn von nun an waren wir ganz auf uns selbst gestellt. Wir waren ausgestattet mit Zielstrebigkeit und einer guten Portion Humor, um den Herausforderungen und ihren Schwierigkeiten entgegenzutreten. ${ }^{7}$

Sie blieben eine Weile in São Paulo und nahmen dann einen Nachtzug in den Bundesstaat Paraná. Die Kaphans hatten noch von Deutschland aus von einer großen englischen Siedlergesellschaft, der Paraná Plantations Ltd. of London, Land gekauft.

Rolândia ist eine Stadt im nördlichen Teil des Bundesstaates Paraná. In den 30er Jahren war es ein Refugium für deutsche Juden, die dem Nazi-Regime entkommen waren (Kosminsky 1985). Die meisten Siedler waren in Deutschland berufstätig gewesen und kamen aus kosmopolitischen Städten wie Berlin, Frankfurt und Hamburg. Die Kunstgalerien und Opernhäuser, an die sie sich erinnerten, kontrastierten merklich mit dem grünen Dschungel, den sie in diesem Teil Brasiliens antrafen. Käte Kaphan erinnert sich ihrer ersten Eindrücke bei ihrer Ankunft mit der Eisenbahn:

Rolândia war die letzte Station auf dieser Strecke. Der Zug war pechschwarz, der Bahnhof dunkel, und jemand brachte uns in das 'Hotel' gegenüber dem Bahnhof, das - Gottseidank - ebenfalls dunkel war (so daß niemand unsere schmutzigen Gesichter sehen konnte). Wir mieteten ein kleines Holzhaus am Hauptplatz. Dort gab es vielleicht 20 oder 30 ähnliche Häuser an der Hauptstraße parallel zu den Eisenbahngleisen, aber diese wurden vom Dschungel verborgen (1).

Bevor ihr Haus fertiggestellt war, lebten sie sieben Monate lang in einem Zelt. Erst nachdem sie das Haus bezogen hatten, widmeten sie sich der Anpflanzung von Nahrungsmitteln und Kaffee. Den ersten Wunsch, eine Kaffee-Plantage anzulegen, erfüllten sie sich mit gemischten Gefühlen, denn das Wachstum der Kaffee-Sträucher war eine riskante Angelegenheit. Man mußte fünf Jahre bis zur ersten Ernte warten, und dies sowie die Möglichkeit, daß ein Nachtfrost alles zerstörte, machte das Unternehmen zu einem Glücksspiel. Aber sie beschlossen, die Chance zu nutzen.

${ }^{7}$ Die Zitate sind dem unveröffentlichten Manuskript entnommen. 
Mit sachkundiger Hilfe rodeten sie den Wald. Hierbei wurden die Bäume gefällt und die Baumstümpfe zum Vermodern zurückgelassen. Das Nutzholz wurde beseitigt, und nach einer wenige Monate dauernden Phase des Trocknens wurde der Wald niedergebrannt. Nach der Brandrodung wurden die Samen ausgesät, und die Bewirtschaftung begann. Der jungfräuliche Boden war fruchtbar und günstig für Mais, Bohnen und Kaffee. Die niedergebrannten Baumstämme wurden als natürlicher Dünger für die Kaffee-Pflanzen an Ort und Stelle gelassen. Jedoch verlief nicht alles nach Plan:

Was wir befürchtet hatten, traf dann auch wirklich ein: Alles wurde in einer einzigen Nacht durch Frost zerstört, genauer noch: durch eine einzige Stunde an einem grauen kalten Morgen mit einer Temperatur von $-4^{\circ}$. Dies warf uns um zwei Jahre zurück. Es geschah nach sechs Jahren in Brasilien, und so brauchten wir acht Jahre bis zur ersten Ernte. Wovon lebten wir in der Zwischenzeit? Wir pflanzten Baumwolle, Reis, Bohnen und hielten vor allem Schweine, weil der Boden fruchtbar genug war für Mais und Maniok. Wir hatten während unserer ersten fünf Jahre eine kleine Weide angelegt - eine Oase in der Wildnis, und hatten so bald wie möglich nach unserer Ankunft zwei Kühe gekauft. (3).

Die Fähigkeit, aus Rückschlägen und Tragödien das Beste zu machen, war charakteristisch für diese Frauen, die nach Brasilien auswanderten. Sie alle waren gebildete Frauen aus der deutschen Mittelschicht, die vor der nationalsozialistischen Machtergreifung gewisse Privilegien und Freiheiten genossen hatten. Sie zogen nach Brasilien aus Notwendigkeit und begegneten dort den Herausforderungen eines in Sprache und Kultur fremden Landes. Marthe Brill, Alice Brill Czapski, Mirjam Logat, Hilde Wiedemann, Trudi Landau und Käte Kaphan beschreiben unterschiedliche Probleme, denen emigrierte Frauen in Brasilien ausgesetzt waren. Aber welche Normen diese Frauen auch immer akzeptierten oder ablehnten, man kann ihr Geschlecht nicht außer Betracht lassen oder als selbstverständlich annehmen. Durch sie erhalten wir ein subjektives Bild von dem, was das Leben für eingewanderte Frauen während dieser schwierigen Zeit war. Details des alltäglichen Lebens beleben die persönliche Sicht der Geschichte.

Die Geschichten entziehen sich jeder traditionellen Kategorisierung und zwingen dazu, von neuem über die Exil-Autobiographien von Frauen nachzudenken. Nicht nur war das Geschlecht ein wichtiger Punkt; die Auswanderung in ein Drittweltland wie Brasilien steigerte noch die Schwierigkeiten, die in Ländern wie den USA oder Kanada vielleicht geringer gewesen wären. Der mit Exil und Emigration verbundene Umbruch zwang Frauen, ihr häusliches Leben zu verlassen und eine durch Übergänge bestimmte Lebensweise anzunehmen, was sich in ihren Memoiren widerspiegelt. Die Frauen schreiben über Aspekte ihres Lebens, die ihr Wesen verändert haben und zwingen dadurch den Leser, über Fragen der Marginalisierung und politisch fixierter Geschlechterrollen 
nachzudenken. Obwohl auf den Seiten Geschichte berichtet wird, geht der Anstoß zum Schreiben von einer persönlichen Geschichte aus, einem Versuch, die Geschichte einer Familie oder einer Gemeinschaft festzuhalten, die fast vollständig untergegangen ist.

Die Texte geben uns einen flüchtigen Einblick in die Gefühle und das Denken deutsch-jüdischer Frauen, die in düstere, von ihnen nicht zu beeinflussende Situationen geraten waren. Sie bieten eine einzigartige Sicht in die weibliche Erfahrung des Krieges, der Entwurzelung und des Exils. Autobiographien von Frauen entsprechen nicht den Erwartungen dessen, was das Leben von Frauen sein sollte, sondern ermöglichen einen seltenen Einblick in die Wahrheit weiblicher Erfahrung.

Aus dem Englischen von Patrik von zur Mühlen

\section{Bibliographie}

Benstock, Shari (Hg.). 1988. The Private Self: Theory and Practice of Women's Autobiographical Writings. Chapel Hill, N.C.: Univ. of North Carolina Press.

Biographisches Handbuch der deutschsprachigen Emigration nach 1933/Interternational Biographical Dictionary of Central European Emigrés 19331945. 1980-83. Hg. von Werner Röder und Herbert A. Strauss. 3 Bde. München: Saur.

Bridenthal, Renate; Atina Grossmann; Marion Kaplan (Hg.). 1984. When Biology Became Destiny. New York: Monthly Review Press.

Brill, Marthe. 1939. Der Schmelztiegel. Manuskript. München: Institut für Zeitgeschichte.

Brill Czapski, Alice. 1991. Memories from 1933-1945. Manuskript. São Paulo: Privatsammlung.

Frühwald, Wolfgang; Wolfgang Schieder (Hg.). 1981. Leben im Exil. Hamburg: Hoffmann \& Campe.

Gruenbaum, Irene. 1950. [ohne Titel] Manuskript. Wien: Dokumentationsarchiv des Österreichischen Widerstands.

Heilbrun, Carolyn G. 1988. Writing a Woman's Life. New York: Ballantine.

Heinemann, Marlene E. 1986. Gender and Destiny. New York: Greenwood.

Kaphan, Käte. 1956. Immigration into the Brazilian Jungle. Manuskript. Rolândia, Paraná: Privatsammlung.

Kießling, Wolfgang. 1981. Exil in Lateinamerika. 2 Bde. Leipzig: Reclam. 
Kosminsky, Ethel Volfzon. 1985. Rolândia, a terra prometida. Judeus refugiados do nazismo no Norte do Paraná. In: Judaica Brasil 4.

Landau, Trudi. 1990. My Life Story. Manuskript. São Paulo: Privatsammlung.

Lesser, Jeff H. 1989. Pawns of the Powerful: Jewish Immigration to Brazil, 1904-1945. New York: Ph.D. Dissertation New York University.

Lixl-Purcell, Andreas. 1988. Women of Exile. New York: Greenwood.

Logat, Mirjam. 1990. Ich bin ausgewandert. Manuskript. São Paulo: Privatsammlung.

Personal Narratives Group. 1989. Interpreting Women's Lives. Bloomington: Indiana UP.

Smith, Sidonie. 1987. A Poetics of Women's Autobiography. Bloomington: Indiana UP.

Wiedemann, Hilde. 1979. Der Weg ist das Ziel. Rio de Janeiro: H. Wiedemann.

Young, James E. 1988. Writing and Rewriting the Holocaust. Bloomington: Indiana UP. 